Pacific Journal of Mathematics

THE SPECTRAL THEOREM FOR UNBOUNDED NORMAL 


\section{THE SPECTRAL THEOREM FOR UNBOUNDED NORMAL OPERATORS}

\section{S. J. BERNAU}

This paper gives a direct constructive proof of the spectral theorem for a normal operator $T$ (bounded or unbounded) in a complex Hilbert space. It depends on the results, recently obtained by elementary methods, that an unbounded positive self adjoint operator $A$ has a unique positive self adjoint square root $A^{1 / 2}$; and an arbitrary self adjoint operator $A$ has a unique representation $A=A^{+}-A^{-}$with $A^{+}$and $A^{-}$self adjoint and positive and the range of each contained in the null space of the other.

Write $|T|=\left(T^{*} T\right)^{1 / 2}$ and, for complex $\lambda$ and $r \geqq 0$, let $E(\lambda, r)$ be the null space projection of $(|T-\lambda I|-r I)^{+}$. For compact subsets $K$ of the complex plane

$$
E(K)=\bigwedge_{c>0} \bigvee\{E(\lambda, \varepsilon): \lambda \in K\}
$$

and for any Borel set $M$,

$$
E(M)=\mathrm{V}\{E(K): K \text { compact and } K \subseteq M\} .
$$

It is shown that $E$ is the unique spectral measure such that

$$
T=\int \lambda E(d \lambda) \text {. }
$$

In the case of a bounded normal operator the spectral theorem can be obtained in many different ways. For example, the theorem can be deduced from the theory of $B^{*}$-algebras [4], the representation of linear functionals on $C(M)$ ( $M$ compact Hausdorff) ([5], [2]), or the Stone-Weierstrass theorem [8]. The proof of the theorem for unbounded normal operators usually relies both on the bounded case and on the theorem for unbounded self adjoint operators [4], [8], [9]).

Our proofs are elementary in the sense of [7]. That is to say we depend only on inherent properties of Hilbert space and of the complex number system. While we use the notation and some elementary results from the theory of spectral measures and integrals these are merely convenient devices for stating the results. Apart from this, and some manipulations with projections, all the results needed are to be found in [1].

The method of proof seems to be new, even in the bounded case. It is motivated, to some extent, by Riesz and Nagy's proof $[8, \S 108]$ 
of the spectral theorem for bounded self-adjoint operators. The proof of uniqueness of the spectral measure of a normal operator is based on the neat characterisation of the spectral subspaces given by Halmos [5, $\S 11]$. This in turn is based on the corresponding results for bounded self adjoint operators given in [7].

To make the paper reasonably self contained statements of the main results of [1] have been included.

I am grateful to the referee for pointing out one serious error and some lesser mistakes in the original manuscript of this paper.

2. Definitions and preliminary results. Throughout this paper $\mathfrak{S}$ denotes a complex Hilbert space. All operators on $\mathscr{S}$ are assumed linear, but not necessarily bounded. For an operator $T, \mathcal{D}(T), \Re(T)$ and $\mathfrak{R}(T)$ denote, respectively, the domain, range and null space of $T$. If $T$ is bounded we assume $\mathfrak{D}(T)=\mathfrak{C}$ and if $T$ is not bounded we assume $\mathfrak{D}(T)$ is dense in $\mathfrak{g}$. By projection we always mean orthogonal projection. All statements about convergence of operators mean strong convergence.

We refer to $[8, \S \S 114-119]$ for definitions and elementary properties of closed operators, the adjoint of an operator and extensions of an operator. Recall that an operator $T$ is self adjoint if $T=T^{*}$, positive if $T$ is self adjoint and $(T x, x) \geqq 0(x \in \mathfrak{D}(T))$; and that $T$ is normal if $T T^{*}=T^{*} T$. If $S$ is bounded we say that $T$ commutes with $S$ if $S T \leqq T S$ (i.e., $T S$ is an extension of $S T$ ).

We record the following theorems.

THEOREM 1. If $A$ is a self adjoint operator there exist unique positive operators $A^{+}$and $A^{-}$such that

$$
A=A^{+}-A^{-}, \mathfrak{R}\left(A^{+}\right) \subseteq \Re\left(A^{-}\right), \Re\left(A^{-}\right) \subseteq \Re\left(A^{+}\right) ;
$$

and $A^{+}$and $A^{-}$commute with every bounded operator which commutes with $A$.

THEOREM 2. If $T$ is a normal operator there exist a positive operator $|T|$ and a unitary operator $U$ such that

$$
T=|T| U=U|T| \text {. }
$$

$|T|=\left(T^{*} T\right)^{1 / 2}$ and is uniquely determined by $T$, and $U$ is unique if we require (as we may) that

$$
U x=x \quad(x \in \mathfrak{N}(T)) .
$$

Furthermore, 


$$
\begin{gathered}
\mathfrak{D}(T)=\mathfrak{D}\left(T^{*}\right)=\mathfrak{D}(|T|), \\
\|T x\|=\left\|T^{*} x\right\|=\||T| x\| \quad(x \in \mathfrak{D}(T)) .
\end{gathered}
$$

For elementary proofs of these theorems see [1, Theorems 12 and 22].

We need some results about suprema and infima of sets of projections. For these we refer to $[5, \S 30]$. We also use the result that a directed increasing (decreasing) set of commuting projections is strongly convergent to its supremum (infimum). A proof of this can be based on [8, §104, p. 263].

Throughout this paper $T$ is a normal, but not necessarily bounded, operator on $\mathfrak{S} ; C$ is the complex plane, $\mathscr{B}$ is the set of all Borel subsets of $C, \mathscr{K}$ is the set of all compact subsets of $C$ and $\mathscr{C}$ is the set of all open subsets of $C$.

3. Construction of the spectral projections. Suppose that $\lambda \in C$ and $r \geqq 0$. The operator $T-\lambda I$ is normal so that $(|T-\lambda I|-r I)^{+}$ is uniquely defined, self adjoint and hence closed. It follows that $\mathfrak{R}\left((|T-\lambda I|-r I)^{+}\right)$is closed. We now define $E(\lambda, r)$ to be the projection on $\mathfrak{R}\left((|T-\lambda I|-r I)^{+}\right) ; E(\lambda, r)$ is a bounded orthogonal projection.

For $K \in \mathscr{K}$ we define

$$
E(K)=\bigwedge_{\varepsilon>0} \bigvee\{E(\lambda, \varepsilon): \lambda \in K\}
$$

and extend the definition of $E$ to arbitrary subsets $M$ of $C$ by the formula,

$$
E(M)=\bigvee\{E(K): K \in \mathscr{K} \text { and } K \leqq M\} .
$$

(Here and subsequently we take the supremum of an empty set of projections to be 0 . This gives $E(\varnothing)=0$.)

In this section we show that $E$ restricted to $\mathscr{B}$ is a spectral measure.

It is important to know that

$$
E(\lambda, r)=E(D(\lambda, r))
$$

where $D(\lambda, r)$ denotes the closed disc with centre $\lambda$ and radius $r$. This result is proved in Lemma 3. Before we can prove (1) we need some commutativity results which apply to all the projections $E(M)$ $(M \subseteq C)$.

Lemma 1. The projections $E(M)(M \subseteq C)$ commute with each 
other and with $T, T^{*}$ and $|T-\lambda I|(\lambda \in C)$.

Proof. As in the proof of [1, Theorem 23] it follows that for each complex $\lambda$ the projections $E(\lambda, r)(r \geqq 0)$ commute with each other and with $T-\lambda I$ and $T^{*}-\bar{\lambda} I\left(=(T-\lambda I)^{*}\right)$. Thus they commute with $T-\mu I$ and $(T-\mu I)^{*}(\mu \in C)$. Hence [1, Theorem 10] they commute with $\left((T-\mu I)^{*}(T-\mu I)^{1 / 2}\right)=|T-\mu I|$ and [1, Lemma 16] with $E(\mu, s)(\mu \in C, s \geqq 0)$. Because multiplication of projections is strongly continuous, infima and suprema of sets of commuting projections are themselves commutative. It follows that

$$
E(M) E(N)=E(N) E(M) \quad(M, N \subseteq C) .
$$

Now let $\mathscr{E}$ be any set of commuting projections all of which commute with $T$. We show that $\bigvee \mathscr{E}$ and $\Lambda \mathscr{E}$ also commute with $T$. Because the projections in $\mathscr{E}$ commute we may, using the formulae for finite suprema of commuting projections, assume that $\mathscr{E}$ is directed increasing. Then $\mathscr{E}$ is strongly convergent to $\bigvee \mathscr{E}$. If $x \in \mathfrak{D}(T)$ and $E \in \mathscr{E}, E x \in \mathfrak{D}(T)$ and $E T x=T E x$. Now, $E x \rightarrow(\bigvee \mathscr{E}) x$ and

$$
T E x=E T x \rightarrow(\bigvee \mathscr{E}) T x .
$$

Because $T$ is closed, $(\mathrm{V} \mathscr{E}) x \in \mathfrak{D}(T)$ and

$$
T(\mathbf{V} \mathscr{E}) x=(\mathrm{V} \mathscr{E}) T x \quad(x \in \mathfrak{D}(T)) .
$$

Thus $(\bigvee \mathscr{E}) T \subseteq T(\bigvee \mathscr{E})$ as required. Similarly $\Lambda \mathscr{E}$ commutes with $T$.

By taking adjoints we deduce that $\bigvee \mathscr{E}$ and $\Lambda \mathscr{E}$ commute with $T^{*}$. It now follows that they commute with $(T-\lambda I)^{*},(T-\lambda \boldsymbol{I})$ and, by [1, Theorem 10] again, with $|T-\lambda I|$. The remainder of the Lemma is now obvious.

Before proving the next lemma we record some known facts about the projections $E(\lambda, r)$.

For fixed $\lambda \in C$ :

$$
\begin{gathered}
E(\lambda, r) \leqq E(\lambda, s) \quad(0 \leqq r<s) ; \\
E(\lambda, r)=\lim _{s \rightarrow r+0} E(\lambda, s) ; \\
E(\lambda, r) \rightarrow I \quad(r \rightarrow \infty) .
\end{gathered}
$$

These are proved in [1, Lemmas 17, 18]. It also follows from [1, Lemma 17 and proof of Theorem 23] that $(T-\lambda I) E(\lambda, r)$ is a bounded normal operator such that

$$
\{(T-\lambda I) E(\lambda, r)\}^{*}=(T-\lambda I)^{*} E(\lambda, r) ;
$$




$$
\|(T-\lambda I) E(\lambda, r)\| \leqq r ;
$$

and, writing $F(\lambda, r)=I-E(\lambda, r)$, that:

$$
(|T-\lambda I| F(\lambda, r) x, F(\lambda, r) x) \geqq r\|F(\lambda, r) x\|^{2} \quad(x \in \mathfrak{D}(T)) .
$$

(Recall that $\mathfrak{D}(|T-\lambda I|)=\mathfrak{D}(T-\lambda I)=\mathfrak{D}(T)(\lambda \in C))$.

It is an immediate corollary of (2) that, for any subset $M$ of $C$ :

$$
\mathrm{V}\{E(\lambda, \varepsilon): \lambda \in M\} \leqq \mathrm{V}\{E(\lambda, \eta): \lambda \in M\} \quad(0 \leqq \varepsilon \leqq \eta) ;
$$

and it follows from the definitions and from elementary properties of suprema and infima that, if $M \subseteq N$,

$$
E(M) \leqq E(N) \text {. }
$$

The next lemma is crucial.

Lemma 2. If $\lambda_{1}, \cdots, \lambda_{n}$ and $\mu$ are complex, $r_{1}, \cdots, r_{n}$ and $r$ are nonnegative and

$$
D(\mu, r) \sqsubseteq \bigcup_{i=1}^{n} D\left(\lambda_{i}, r_{i}\right)
$$

then

$$
E(\mu, r) \leqq \bigvee_{i=1}^{n} E\left(\lambda_{i}, r_{i}\right)
$$

Proof. Take $\varepsilon>0$ and write

$$
\begin{gathered}
F_{i}=E\left(\lambda_{i}, r_{i}+\varepsilon\right), \\
F_{\varepsilon}=\prod_{i=1}^{n}\left(E(\mu, r)-E(\mu, r) F_{i}\right) .
\end{gathered}
$$

Because all the projections commute, $F_{\varepsilon}$ is a projection,

$$
F_{\varepsilon}=E(\mu, r)-E(\mu, r) \bigvee_{i=1}^{n} F_{i},
$$

and, because all the $E(\lambda, r)$ commute with $T$ (see proof of Lemma 1), $F_{\varepsilon}$ commutes with $T$.

By $(6),(T-\mu I) F_{\varepsilon}$ is a bounded normal operator and $\left\|(T-\mu I) F_{\varepsilon}\right\| \leqq r$. For the remainder of the proof we write $F$ for $F_{\varepsilon}$ and we assume, as we may, that $\mu=0$. By [2, Theorem 2], because $T F$ is bounded and normal, there exist a complex number $\alpha$ and a sequence $\left(x_{k}\right)$ in $\mathfrak{S}$ such that: $|\alpha|=\|T F\|,\left\|x_{k}\right\|=1$ for all $k$ and

$$
T F x_{k}-\alpha x_{k} \rightarrow 0 \quad(k \rightarrow \infty) .
$$

Now, because $T$ commutes with $F, F T F=T F$ and hence, 


$$
\alpha\left(x_{k}-F x_{k}\right)=(F-I)\left(T F x_{k}-\alpha x_{k}\right) \rightarrow 0 \quad(k \rightarrow \infty) .
$$

Suppose that $\alpha \neq 0$, then

$$
x_{k}-F x_{k} \rightarrow 0 \quad(k \rightarrow \infty) .
$$

Consequently $\left\|F x_{k}\right\| \rightarrow 1(k \rightarrow \infty)$ and we may, and do, assume that $x_{k}=F x_{k}$ for all $k$.

Now, for $i=1,2, \cdots, n$,

$$
\begin{aligned}
\left|\alpha-\lambda_{i}\right| & =\left\|\left(\alpha-\lambda_{i}\right) F x_{k}\right\| \\
& =\left\|\left(T-\lambda_{i} I\right) F x_{k}-(T-\alpha I) F x_{k}\right\| \\
& \geqq\left\|\left(T-\lambda_{i} I\right) F x_{k}\right\|-\left\|(T-\alpha I) F x_{k}\right\| .
\end{aligned}
$$

Because $F=\left(I-F_{i}\right) F$, it follows from Theorem 2 and (7) that

$$
\begin{aligned}
\left\|\left(T-\lambda_{i} I\right) F x_{k}\right\| & =\left\|\left|\left(T-\lambda_{i} I\right)\right| F x_{k}\right\|\left\|F x_{k}\right\| \\
& \geqq\left(\left|T-\lambda_{i} I\right| F x_{k}, F x_{k}\right) \\
& \geqq\left(r_{i}+\varepsilon\right)\left\|F x_{k}\right\|^{2} \\
& =r_{i}+\varepsilon .
\end{aligned}
$$

Hence,

$$
\left|\alpha-\lambda_{i}\right| \geqq\left(r_{i}+\varepsilon\right)-\left\|(T-\alpha I) F x_{k}\right\| \rightarrow r_{i}+\varepsilon \quad(k \rightarrow \infty) .
$$

Because $|\alpha|=\|T F\| \leqq r$ and $D(0, r) \leqq \bigcup D\left(\lambda_{i}, r_{i}\right)$, we have $\left|\alpha-\lambda_{i}\right| \leqq r_{i}$ for some $i$. This is a contradiction so we must have $\alpha=0$, i.e. $T F=0$. Again, for some $i, 0 \in D\left(\lambda_{i}, r_{i}\right)$ and, as above,

$$
\begin{aligned}
\left(r_{i}+\varepsilon\right)\|F x\|^{2} & \leqq\left\|\left(T-\lambda_{i} I\right) F x\right\|\|F x\| \\
& =\left\|-\lambda_{i} F x\right\|\|F x\| \\
& =\left|\lambda_{i}\right|\|F x\|^{2} \quad(x \in \mathfrak{S}) .
\end{aligned}
$$

Because $0 \in D\left(\lambda_{i}, r_{i}\right),\left|\lambda_{i}\right| \leqq r_{i}$. Hence $\|F x\|=0(x \in \mathfrak{S})$ and $F=0$.

Now let $\varepsilon \rightarrow 0+0$, by (3), $F_{i} \rightarrow E\left(\lambda_{i}, r_{i}\right)$ for each $\underline{i}$. Thus, because multiplication of projections is strongly continuous,

$$
\begin{aligned}
0=F_{\varepsilon} & \rightarrow \prod_{i=1}^{n}\left(E(\mu, r)-E(\mu, r) E\left(\lambda_{i}, r_{i}\right)\right) \\
& =E(\mu, r)-E(\mu, r) \bigvee_{i=1}^{n} E\left(\lambda_{i}, r_{i}\right) ;
\end{aligned}
$$

and $E(\mu, r) \leqq \bigvee_{i=1}^{n} E\left(\lambda_{i}, r_{i}\right)$, as required.

Lemma 3. For $\lambda \in C, r \geqq 0$,

$$
E(\mu, r)=\bigwedge_{\varepsilon>0} \bigvee\{E(\lambda, \varepsilon): \lambda \in D(\mu, r)\}
$$


Proof. If $\eta>0$, it follows from Lemma 2 that

$$
E(\mu, r+\eta) \geqq E(\lambda, \varepsilon) \quad(\lambda \in D(\mu, r) ; 0<\varepsilon \leqq \eta) .
$$

Hence

$$
E(\mu, r+\eta) \geqq \underset{\varepsilon>0}{\Lambda} \bigvee\{E(\lambda, \varepsilon): \lambda \in D(\mu, r)\} ;
$$

and by (3),

$$
E(\mu, r) \geqq \bigwedge_{\varepsilon>0} \bigvee\{E(\lambda, \varepsilon): \lambda \in D(\mu, r)\} .
$$

Conversely, for each $\varepsilon>0$, the set of open $\operatorname{discs}\{z:\|z-\lambda\|<\varepsilon\}$ $(\lambda \in D(\mu, r))$ covers the compact set $D(\mu, r)$. Hence, there exist $\lambda_{1}, \cdots, \lambda_{n}$ such that

$$
D(\mu, r) \subseteq \bigcup_{i=1}^{n} D\left(\lambda_{i}, \varepsilon\right)
$$

By Lemma 2,

$$
\begin{aligned}
E(\mu, r) & \leqq \bigvee_{i=1}^{n} E\left(\lambda_{i}, \varepsilon\right) \\
& \leqq \bigvee\{E(\lambda, \varepsilon): \lambda \in D(\mu, r)\} .
\end{aligned}
$$

Thus

$$
E(\mu, r) \leqq \bigwedge_{\varepsilon>0} \bigvee\{E(\lambda, \varepsilon): \lambda \in D(\mu, r)\}
$$

and the proof is complete.

LeMMA 4. If $K$ and $L$ are compact,

$$
E(K) \vee E(L)=E(K \cup L) \text {. }
$$

Proof. Because all the projections commute, it follows from [5, $\S 30$, Theorem 3] that

$$
\begin{aligned}
E(K) & \vee E(L) \\
& =\left[\bigwedge_{\varepsilon>0} \bigvee\{E(\lambda, \varepsilon): \lambda \in K\}\right] \vee\left[\bigwedge_{\eta>0} \mathrm{~V}\{E(\mu, \eta): \mu \in L\}\right] \\
& =\bigwedge_{\varepsilon, \eta>0}([\mathrm{~V}\{E(\lambda, \varepsilon): \lambda \in K\}] \vee[\bigvee\{E(\mu, \eta): \mu \in L\}]) \\
& =\bigwedge_{\varepsilon, \eta>0} \mathrm{~V}\{E(\lambda, \varepsilon) \vee E(\mu, \eta): \lambda \in K, \mu \in L\} .
\end{aligned}
$$

Hence, by (8), 


$$
\begin{aligned}
E(K) \vee E(L) & =\bigwedge_{\varepsilon>0} \bigvee\{E(\lambda, \varepsilon) \vee E(\mu, \varepsilon): \lambda \in K, \mu \in L\} \\
& =\bigwedge_{\varepsilon>0} \bigvee\{E(\nu, \varepsilon): \nu \in K \cup L\} \\
& =E(K \cup L) .
\end{aligned}
$$

Lemma 5. If $M$ and $N$ are disjoint subsets of $C$ then,

$$
E(M) E(N)=0 \text {. }
$$

Proof. Let $K$ and $L$ be compact subsets of $M$ and $N$ respectively. Then $K \cap L=\varnothing$ and hence there exists a positive $\eta$ such that

$$
D(\lambda, \eta) \cap D(\mu, \eta)=\varnothing \quad(\lambda \in K, \mu \in L) .
$$

Because all the relevant projections commute it is sufficient now to prove that $E(\lambda, \eta) E(\mu, \eta)=0(\lambda \in K, \mu \in L)$. Let $x \in \mathfrak{S}$ and write

$$
y=E(\lambda, \eta) E(\mu, \eta) x \text {. }
$$

Because $y=E(\lambda, \eta) y=E(\mu, \eta) y$, it follows from (6) that,

$$
\begin{aligned}
\|(\lambda-\mu) y\| & =\|(T-\mu I) E(\mu, \eta) y-(T-\lambda I) E(\lambda, \eta) y\| \\
& \leqq\|(T-\mu I) E(\mu, \eta) y\|+\|(T-\lambda I) E(\lambda, \eta) y\| \\
& \leqq \eta\|y\|+\eta\|y\| .
\end{aligned}
$$

Because $D(\lambda, \eta) \cap D(\mu, \eta)=\varnothing,|\lambda-\mu|>2 \eta$. Hence $\|y\|=0$ and $E(\lambda, \eta) E(\mu, \eta)=0$ as required.

Corollary. If $K$ and $L$ are in $\mathscr{C}$ and $K \cap L=\varnothing$,

$$
E(K \cup L)=E(K)+E(L) \text {. }
$$

Proof. This follows from Lemmas 4 and 5 .

Lemma 6. If $K$ is in $\mathscr{K}$

$$
E(K)=\Lambda\{E(U): U \in \mathscr{U}, K \subseteq U\} .
$$

Proof. By definition of $E$,

$$
E(K) \leqq \Lambda\{E(U): U \in \mathscr{\ell}, K \leqq U\} .
$$

To prove the converse let

$$
E_{\varepsilon}=\bigvee\{E(\lambda, \varepsilon): \lambda \in K\} \quad(\varepsilon>0) .
$$

By definition, $E(K)=\Lambda_{\varepsilon>0} E_{\varepsilon}$. Let $U_{\varepsilon}$ be the open $\varepsilon$-neighbourhood of $K$; i.e. 


$$
U_{\varepsilon}=\{z \in C: d(z, K)<\varepsilon\} \quad(\varepsilon>0) .
$$

Clearly $K \leqq U_{\varepsilon}$. We complete the proof by showing that $E\left(U_{\varepsilon}\right) \leqq E_{\varepsilon}$ $(\varepsilon>0)$.

Suppose that $L \in \mathscr{K}$ and $L \cong U_{\varepsilon}$. Then $L$ is at positive distance from $C \sim U_{\varepsilon}$, i.e. there exists $\eta$ such that $\eta>0$ and if $\mu \in L$ and $|z-\mu| \leqq \eta$ then $z \in U_{\varepsilon}$. Thus, for each $\mu$ in $L$, the compact set $D(\mu, \eta)$ is covered by the open discs $\{z:|z-\lambda|<\varepsilon\}(\lambda \in K)$. Hence a finite set, corresponding, say, to $\lambda_{1}, \cdots, \lambda_{n}$, of these dises cover $D(\mu, \eta)$. Then, by Lemma 2 ,

$$
\begin{aligned}
E(\mu, \eta) & \leqq \bigvee_{i=1}^{n} E\left(\lambda_{i}, \varepsilon\right) \\
& \leqq E_{\varepsilon} \quad(\mu \in L) .
\end{aligned}
$$

Thus $E(L) \leqq E_{\varepsilon}\left(L \in \mathscr{K}\right.$ and $\left.L \leqq U_{\varepsilon}\right)$ and hence, $E\left(U_{\varepsilon}\right) \leqq E_{\varepsilon}$. It follows that

$$
E(K) \leqq \bigwedge_{\varepsilon>0} E\left(U_{\varepsilon}\right) \leqq \bigwedge_{\varepsilon>0} E_{\varepsilon}=E(K),
$$

and, because each $U_{\varepsilon}$ is open,

$$
E(K)=\bigwedge\{E(U): U \in \mathscr{\ell}, K \subseteq U\} .
$$

At this stage it is relevant to point out that we have proved enough to show that, for each $x$ in $\mathfrak{S}$, the function $(E() x, x)$ restricted to $\mathscr{\mathcal { C }}$ is a regular content. Standard techniques $[6, \S \S 53,54]$ would enable us to extend this content to a regular Borel measure. We would then have to show that this measure coincided with the restriction of $(E() x, x)$ to $\mathscr{B}$. It would then follow $[5, \S 36]$ that $E$ restricted to $\mathscr{B}$ was a spectral measure. We do not proceed in this way because the proof that $(E() x, x)$ was the extension of the content originally defined would be of the same order of magnitude as the direct proof that $E$ restricted to $\mathscr{B}$ is a spectral measure. There are, however, obvious similarities between our proofs and the standard procedures for extending a content.

Let $\mathscr{A}$ denote the class of all subsets $M$ of $C$ such that

$$
E(M)=\bigwedge\{E(U): U \in \mathscr{Q}, M \subseteq U\} .
$$

Clearly $\mathscr{K} \subseteq \mathscr{A}$ and $\mathscr{Q} \subseteq \mathscr{A}$. We shall show that $\mathscr{B} \subseteq \mathscr{A}$ and that $E$ restricted to $\mathscr{A}$ (and hence, restricted to $\mathscr{B}$ ) is a spectral measure.

Lemma 7. If $\left(U_{n}\right)$ is a sequence in $\mathscr{Q}$ and $U=\bigcup_{n=1}^{\infty} U_{n}$, then

$$
E(U)=\bigvee_{n=1}^{\infty} E\left(U_{n}\right)
$$


Proof. By (9), $E(U) \geqq \mathrm{V}_{n=1}^{\infty} E\left(U_{n}\right)$.

Conversely let $K \in \mathscr{K}, K \subseteq U$. Because $K$ is compact there exists $m$ such that

$$
K \subseteq \bigcup_{n=1}^{m} U_{n}
$$

Hence, $\left[6, \S 50\right.$, Theorem A], there exist compact $K_{1}, \cdots, K_{m}$ such that

$$
\begin{aligned}
& K_{n} \subseteq U_{n} \quad(n=1, \cdots, m), \\
& K=\bigcup_{n=1}^{m} K_{n} .
\end{aligned}
$$

Then, by definition, $E\left(K_{n}\right) \leqq E\left(U_{n}\right)$ for each $n$ and, by Lemma 4 ,

$$
\begin{aligned}
E(K) & =\bigvee_{n=1}^{m} E\left(K_{n}\right) \\
& \leqq \bigvee_{n=1}^{m} E\left(U_{n}\right) \\
& \leqq \bigvee_{n=1}^{\infty} E\left(U_{n}\right) .
\end{aligned}
$$

Thus $E(U) \leqq \bigvee_{n=1}^{\infty} E\left(U_{n}\right)$, which completes the proof.

Lemma 8. If $\left(M_{n}\right)$ is a sequence in $\mathscr{A}$ and $M=\bigcup_{n=1}^{\infty} M_{n}$, then $M$ is in $\mathscr{A}$ and

$$
E(M)=\bigvee_{n=1}^{\infty} E\left(M_{n}\right)
$$

Proof. By (9), $E(M) \geqq \bigvee_{n=1}^{\infty} E\left(M_{n}\right)$.

Now, suppose that $\varepsilon>0$ and $x \in \mathfrak{S}$. By definition of $\mathscr{A}$ there exists a sequence $\left(U_{n}\right)$ in $\mathscr{U}$ such that $M_{n} \subseteq U_{n}$ and,

$$
\left\|E\left(U_{n}\right) x-E\left(M_{n}\right) x\right\|<\varepsilon 2^{-n} \quad(n=1,2, \cdots) .
$$

Let $U=\bigcup_{n=1}^{\infty} U_{n} ; U$ is open, $M \subseteq U$ and, by (9) and Lemma 7,

$$
E(M) \leqq E(U)=\bigvee_{n=1}^{\infty} E\left(U_{n}\right)
$$

Thus,

$$
\begin{aligned}
0 \leqq E(M)-\mathrm{V} E\left(M_{n}\right) & \leqq \mathrm{V} E\left(U_{n}\right)-\mathrm{V} E\left(M_{n}\right) \\
& \leqq \mathrm{V}\left(E\left(U_{n}\right)-E\left(M_{n}\right)\right)
\end{aligned}
$$

Hence, 


$$
\begin{aligned}
\left\|E(M) x-\left(\mathbf{V} E\left(M_{n}\right)\right) x\right\| & \leqq\left\|\left\{\mathbf{V}\left(E\left(U_{n}\right)-E\left(M_{n}\right)\right)\right\} x\right\| \\
& \leqq \sum_{n=1}^{\infty}\left\|E\left(U_{n}\right) x-E\left(M_{n}\right) x\right\| \\
& <\sum_{n=1}^{\infty} \varepsilon 2^{-n} \\
& =\varepsilon .
\end{aligned}
$$

Thus $E(M) x=\left(\bigvee_{n=1}^{\infty} E\left(M_{n}\right)\right) x(x \in \mathfrak{C})$ and $E(M)=\mathrm{V} E\left(M_{n}\right)$.

It also follows from the proof above that

$$
\inf \{\|E(U) x-E(M) x\|: U \in \mathscr{U}, M \subseteq U\}=0 \quad(x \in \mathfrak{S}) .
$$

Hence,

$$
E(M)=\Lambda\{E(U): U \in \mathscr{U}, M \subseteq U\},
$$

$M \in \mathscr{A}$ and the proof is complete.

Corollary 1. If $\left(M_{n}\right)$ is a disjoint sequence in $\mathscr{A}$,

$$
E\left(\bigcup_{n=1}^{\infty} M_{n}\right)=\sum_{n=1}^{\infty} E\left(M_{n}\right)
$$

with the series strongly convergent.

Proof. By Lemma 5,

$$
\bigvee_{n=1}^{\infty} E\left(M_{n}\right)=\sum_{n=1}^{\infty} E\left(M_{n}\right),
$$

with the series strongly convergent.

Corollary 2. Every closed subset of $C$ is in $\mathscr{A}$.

Proof. Every closed subset of $C$ is a countable union of compact sets.

Lemma 9. $E(C)=I$ and, for every $M$ in $\mathscr{A}$, if $M^{\prime}=C \sim M$, then $M^{\prime} \in \mathscr{A}$ and $E\left(M^{\prime}\right)=I-E(M)$.

Proof. Because $C$ is open, $C \in \mathscr{A}$ and, by (9) and (4),

$$
E(C) \geqq E(0, r) \rightarrow I \quad(r \rightarrow \infty) .
$$

If $U$ is open, $U^{\prime}$ is closed and, by the corollaries to Lemma 8,

$$
\begin{aligned}
E\left(U^{\prime}\right)+E(U) & =E(C), \\
E\left(U^{\prime}\right) & =I-E(U) .
\end{aligned}
$$


Similarly, if $K$ is compact, $K^{\prime}$ is open and

$$
E\left(K^{\prime}\right)=I-E(K) \text {. }
$$

Thus, by (9),

$$
\begin{aligned}
E\left(M^{\prime}\right) & \geqq \bigvee\left\{E\left(U^{\prime}\right): U \in \mathscr{C}, M \leqq U\right\} \\
& =\bigvee\{I-E(U): U \in \mathscr{U}, M \leqq U\} \\
& =I-\Lambda\{E(U): U \in \mathscr{U}, M \leqq U\} \\
& =I-E(M) ;
\end{aligned}
$$

and conversely,

$$
\begin{aligned}
& E\left(M^{\prime}\right) \leqq \Lambda\left\{E\left(K^{\prime}\right): K \in \mathscr{C}, K \subseteq M\right\} \\
& =\Lambda\{I-E(K): K \in \mathscr{K}, K \cong M\} \\
& =I-\mathrm{V}\{E(K): K \in \mathscr{K}, K \subseteq M\} \\
& =I-E(M) \text {. }
\end{aligned}
$$

It follows that $E\left(M^{\prime}\right)=I-E(M)$ and, because $K^{\prime} \in \mathscr{U}$ if $K \in \mathscr{K}$, the second inequality above shows that $M^{\prime} \in \mathscr{A}$.

THEOREM 3. If $E$ is restricted to $\mathscr{B}$ then $E$ is a spectral measure.

Proof. Lemmas 8 and 9 show that $\mathscr{A}$ is a $\sigma$-ring of subsets of $C$. Because, $\mathscr{K} \cong \mathscr{A}$, it follows that $\mathscr{B} \cong \mathscr{A}$. Because $E$ is (strongly) countably additive on $\mathscr{A}$ (Lemma 8, Corollary 1) and $E(C)=I$ (Lemma 9), it follows that $E$, restricted to $\mathscr{B}$, is a spectral measure.

REMARK. The proof given above shows that the spectral measure given by the restriction of $E$ is regular, i.e. if $M$ is in $\mathscr{B}$,

$$
\begin{aligned}
E(M) & =\bigvee\{E(K): K \in \mathscr{K}, K \leqq M\} \\
& =\Lambda\{E(U): U \in \mathscr{\ell}, M \leqq U\} .
\end{aligned}
$$

The proof can easily be adapted to give a simple direct proof that a complex spectral measure $[5, \S 39]$ is regular.

4. The spectral theorem. We now wish to prove the relation

$$
T=\int \lambda E(d \lambda)
$$

Before doing this we digress to define spectral integrals and recall some elementary facts about them. Our remarks are based on [4, 
XII. 2.5] and [5, §37].

If $f$ is a complex-valued Borel-measurable function defined on $\underline{C}$ and $r>0, f_{r}$ is defined by

$$
\begin{array}{ll}
f_{r}(\lambda)=f(\lambda) & (|f(\lambda)| \leqq r), \\
f_{r}(\lambda)=0 & (|f(\lambda)|>r) .
\end{array}
$$

If $E$ is any spectral measure we define

$$
A=\int f(\lambda) E(d \lambda)
$$

as follows. $\mathfrak{D}(A)$ is the set of all $x$ in $\mathfrak{S}$ such that $\int f_{r}(\lambda) E(d \lambda) x$ tends to a limit as $r \rightarrow \infty$; and, for $x$ in $\mathfrak{D}(A)$

$$
A x=\lim _{r \rightarrow \infty} \int f_{r}(\lambda) E(d \lambda) x .
$$

(We make the convention that the range of integration is the whole of $C$ unless otherwise specified). Writing $A_{r}=\int f_{r}(\lambda) E(d \lambda)$ and

$$
M_{r}=\{\lambda \in C:|f(\lambda)| \leqq r\},
$$

we have, for $x$ in $\mathfrak{S}$,

$$
\begin{aligned}
\left\|A_{r} x-A_{s} x\right\|^{2} & =\int\left|f_{r}(\lambda)-f_{s}(\lambda)\right|^{2}(E(d \lambda) x, x) \\
& =\int_{M_{r^{\Delta M}}}|f(\lambda)|^{2}(E(d \lambda) x, x) .
\end{aligned}
$$

It follows that

$$
\mathfrak{D}(A)=\left\{x \in \mathfrak{S}: \int|f(\lambda)|^{2}(E(d \lambda) x, x)<\infty\right\},
$$

and

$$
\|A x\|^{2}=\int|f(\lambda)|^{2}(E(d \lambda) x, x) \quad(x \in \mathfrak{D}(A)) .
$$

For the remainder of this paper $E$ denotes the spectral measure with domain $\mathscr{B}$ which we obtained in $\S 3$ (Theorem 3).

THEOREM 4. $T=\int \lambda E(d \lambda)$.

Proof. If $M$ is a Borel set of diameter not greater than $r$ and if $\lambda \in M$, it follows from (5) and (6) that

$$
E(M) x=E(M) E(\lambda, r) x \in \mathfrak{D}(T)
$$


and

$$
\|T E(M) x-\lambda E(M) x\| \leqq r\|E(M) x\| \quad(x \in \mathfrak{S})
$$

Hence

$$
\begin{aligned}
\int_{|\lambda| \leqq r} \lambda E(d \lambda) x & =\int_{|\lambda| \leqq r} \lambda E(d \lambda) E(0, r) x \\
& =T E(0, r) x \quad(x \in \mathfrak{S}) .
\end{aligned}
$$

Thus, if $x \in \mathfrak{D}(T)$,

$$
\begin{aligned}
\int_{|\lambda| \leq r} \lambda E(d \lambda) x & =T E(0, r) x \\
& =E(0, r) T x \\
& \rightarrow T x \quad(r \rightarrow \infty) .
\end{aligned}
$$

This shows that $x \in \mathfrak{D}\left(\int \lambda E(d \lambda)\right)$ and

$$
\int \lambda E(d \lambda) x=T x
$$

so that $T \subseteq \int \lambda E(d \lambda)$. On the other hand if $x \in \mathfrak{D}\left(\int \lambda E(d \lambda)\right)$,

$$
\begin{aligned}
T E(0, r) x & =\int_{|\lambda| \leqq r} \lambda E(d \lambda) x \\
& \rightarrow \int \lambda E(d \lambda) x \quad(r \rightarrow \infty) .
\end{aligned}
$$

Because $T$ is closed and $E(0, r) x \rightarrow x(r \rightarrow \infty)$, we have $x \in \mathfrak{D}(T)$ and $T x=\int \lambda E(d \lambda) x$. Thus $T=\int \lambda E(d \lambda)$ as required.

The construction of $E$ makes uniqueness easy to prove.

THEOREM 5. If $F$ is a spectral measure (with domain $\mathscr{B}$ ) and $T=\int \lambda F(d \lambda)$, then $E=E$.

Proof. Suppose that $\lambda \in C$ and $r \geqq 0$. Let $\mathfrak{F}(\lambda, r)$ be the set of all $x$ in $\mathfrak{S}$ such that

$$
x \in \mathfrak{D}\left(T^{n}\right) \text { and }\left\|r^{-n}(T-\lambda I)^{n} x\right\| \leqq\|x\| \quad(n=1,2, \cdots) .
$$

The proof of $[5, \S 41$, Theorem 1] shows that $\mathfrak{F}(\lambda, r)$ is a subspace of $\mathfrak{S}$ which is invariant under every bounded operator which commutes with $T$. We show that

$$
\Re(F(D(\lambda, r)))=\mathfrak{F}(\lambda, r)=\Re(E(\lambda, r)) .
$$

Write $F(\lambda, r)=F(D(\lambda, r))$. Because $D(\lambda, r)$ is bounded 


$$
\left.F(\lambda, r) x \in \mathfrak{D}\left(T^{n}\right)=\mathfrak{D}(T-\lambda I)^{n}\right) \quad(x \in \mathfrak{S})
$$

and, by (10),

$$
\begin{aligned}
\| r^{-n} & (T-\lambda I)^{n} F(\lambda, r) x \|^{2} \\
& =\int^{-2 n}|\mu-\lambda|^{2 n}(F(d \mu) F(\lambda, r) x, F(\lambda, r) x) \\
& =\int_{D(\lambda, r)} r^{-2 n}|\mu-\lambda|^{2 n}(F(d \mu) F(\lambda, r) x, x) \\
& \leqq \\
& =\|F(\lambda, r) x\|^{2} .
\end{aligned}
$$

Thus $\mathfrak{R}(F(\lambda, r)) \subseteq \mathfrak{F}(\lambda, r)$.

Now suppose that $x \in \mathfrak{F}(\lambda, r)$. Take $s>r$ and write $y=x-F(\lambda, s) x$. Because $\mathfrak{F}(\lambda, r)$ is invariant under $F(\lambda, s), y \in \mathfrak{F}(\lambda, r)$ and $\|(T-\lambda I) y\| \leqq r\|y\|$. Also,

$$
\|(T-\lambda I) y\|^{2}=\int|\mu-\lambda|^{2}(F(d \mu) y, y) ;
$$

and, because $F(\lambda, s) y=0$,

$$
\begin{aligned}
\|(T-\lambda I) y\|^{2} & =\int_{|\mu-\lambda|>s}|\mu-\lambda|^{2}(F(d \mu) y, y) \\
& \geqq s^{2} \int(F(d \mu) y, y) \\
& =s^{2}\|y\|^{2} .
\end{aligned}
$$

Thus, because $s>r,\|y\|=0$. Accordingly, $F(\lambda . s) x=x(x \in \mathfrak{F}(\lambda, r))$. Letting $s \rightarrow r+0$, we have

$$
x=F(\lambda, r) x \quad(x \in \mathfrak{F}(\lambda, r)) .
$$

Thus $\mathfrak{R}(F(\lambda, r))=\mathfrak{F}(\lambda, r)$.

A similar argument, shows that $\mathfrak{R}(E(\lambda, r))=\mathfrak{F}(\lambda, r)$.

Thus the spectral measures $E$ and $F$ agree on all closed dises $D(\lambda, r)$ $(\lambda \in C, r \geqq 0)$. Hence they agree on the $\sigma$-ring generated by these dises, i.e., on $\mathscr{B}$. Thus $E=F$ as required.

We now define the spectral measure (or resolution of the identity) of a normal operator $T$ to be the unique spectral measure $E$ such that

$$
T=\int \lambda E(d \lambda)
$$

We conclude with the important commutativity result. 
THEOREM 6. If $T$ is normal its spectral measure commutes with every bounded operator which commutes with $T$.

(i.e. If $B$ is bounded and $B T \subseteq T B$ then $B E(M)=E(M) B(M \in \mathscr{B})$.)

Proof. For each complex $\lambda$ and nonnegative $r, \mathfrak{F}(\lambda, r)$ is invariant under $B$. Because $\mathfrak{F}(\lambda, r)=\Re(E(\lambda, r)), B E(\lambda, r)=E(\lambda, r) B E(\lambda, r)$. Because $B$ commutes with $T, B^{*}$ commutes with $T^{*}$ and, because

$$
\|(T-\lambda I) x\|=\left\|\left(T^{*}-\bar{\lambda} I\right) x\right\|(x \in \mathfrak{D}(T)),
$$

it follows that $\mathfrak{F}(\lambda, r)$ is invariant under $B^{*}$ so that $B^{*} E(\lambda, r)=$ $E(\lambda, r) B^{*} E(\lambda, r)$ and, finally, $B E(\lambda, r)=E(\lambda, r) B$. The desired result is now an immediate consequence of the construction of $E$.

\section{REFERENCES}

1. S. J. Bernau, The square root of a positive self adjoint operator, (to appear).

2. The spectral theorem for normal operators, J. London Math. Soc. 40 (1965), 478-86.

3. S. J. Bernau and F. Smithies, A note on normal operators, Proc. Cambridge Phil. Soc. 59 (1963), 727-29.

4. N. Dunford and J. T. Schwartz, Linear Operators, Part II, Interscience, New York, 1963.

5. P. R. Halmos, Introduction to Hilbert space and the theory of Spectral Multiplicity, 2nd edition, Chelsea, New York, 1957.

6. - Measure Theory, Van Nostrand, New York, 1959.

7. B. A. Lengyel and M. H. Stone, Elementary proof of the spectral theorem, Ann. of Math. (2) 37 (1936), 853-64.

8. F. Riesz and B. Sz. Nagy, Functional Analysis, Ungar, New York, 1955.

9. M. H. Stone, Linear transformations in Hilbert space and their applications to analysis, Amer. Math. Soc. Colloquium Publications, Vol. XV, New York, 1932.

Received November 4, 1965.

UNIVERSITY OF OTAGO

Dunedin, NeW ZeALAND 


\section{PACIFIC JOURNAL OF MATHEMATICS}

\section{EDITORS}

H. SAMELSON

Stanford University

Stanford, California

J. P. JANS

University of Washington

Seattle, Washington 98105

\section{J. DuGUNDJI}

University of Southern California

Los Angeles, California 90007

RICHARD ARENS

University of California

Los Angeles, California 90024

\section{ASSOCIATE EDITORS}

E. F. BECKENBACH
B. H. NEUMANN

F. WOLF

K. YoSIDA

\section{SUPPORTING INSTITUTIONS}

\author{
UNIVERSITY OF BRITISH COLUMBIA \\ CALIFORNIA INSTITUTE OF TECHNOLOGY \\ UNIVERSITY OF CALIFORNIA \\ MONTANA STATE UNIVERSITY \\ UNIVERSITY OF NEVADA \\ NEW MEXICO STATE UNIVERSITY \\ OREGON STATE UNIVERSITY \\ UNIVERSITY OF OREGON \\ OSAKA UNIVERSITY \\ UNIVERSITY OF SOUTHERN CALIFORNIA
}

\author{
STANFORD UNIVERSITY \\ UNIVERSITY OF TOKYO \\ UNIVERSITY OF UTAH \\ WASHINGTON STATE UNIVERSITY \\ UNIVERSITY OF WASHINGTON \\ AMERICAN MATHEMATICAL SOCIETY \\ CHEVRON RESEARCH CORPORATION \\ TRW SYSTEMS \\ NAVAL ORDNANCE TEST STATION
}

Mathematical papers intended for publication in the Pacific Journal of Mathematics should be typewritten (double spaced). The first paragraph or two must be capable of being used separately as a synopsis of the entire paper. It should not contain references to the bibliography. Manuscripts may be sent to any one of the four editors. All other communications to the editors should be addressed to the managing editor, Richard Arens at the University of California, Los Angeles, California 90024 .

50 reprints per author of each article are furnished free of charge; additional copies may be obtained at cost in multiples of 50 .

The Pacific Journal of Mathematics is published monthly. Effective with Volume 16 the price per volume (3 numbers) is $\$ 8.00$; single issues, $\$ 3.00$. Special price for current issues to individual faculty members of supporting institutions and to individual members of the American Mathematical Society: $\$ 4.00$ per volume; single issues $\$ 1.50$. Back numbers are available.

Subscriptions, orders for back numbers, and changes of address should be sent to Pacific Journal of Mathematics, 103 Highland Boulevard, Berkeley 8, California.

Printed at Kokusai Bunken Insatsusha (International Academic Printing Co., Ltd.), No. 6, 2-chome, Fujimi-cho, Chiyoda-ku, Tokyo, Japan.

\section{PUBLISHED BY PACIFIC JOURNAL OF MATHEMATICS, A NON-PROFIT CORPORATION}

The Supporting Institutions listed above contribute to the cost of publication of this Journal, but they are not owners or publishers and have no responsibility for its content or policies. 


\section{Pacific Journal of Mathematics

Vol. 19, No. $3 \quad$ July, 1966

S. J. Bernau, The spectral theorem for unbounded normal operators .......

Lu-san Chen, Asymptotic behavior of solutions of parabolic equations of

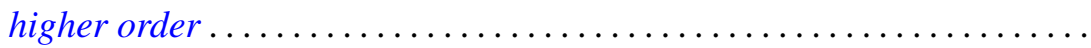

Lawrence William Conlon, An application of the Bott suspension map to the

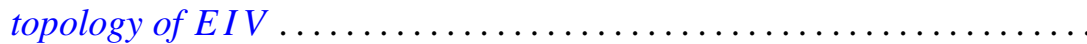

Neal Eugene Foland and John M. Marr, Sets with zero-dimensional kernels .........................................

Stanley Phillip Franklin and R. H. Sorgenfrey, Closed and image-closed

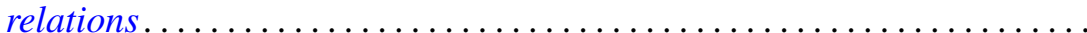

William Jesse Gray, A note on topological transformation groups with a

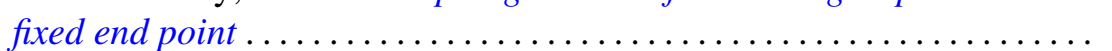

Myron Goldstein, $K$ - and L-kernels on an arbitrary Riemann surface ...... 449

George Joseph Kertz and Francis Regan, The exponential analogue of a generalized Weierstrass series .............................

Walter Leighton, On Liapunov functions with a single critical point ........ 467

Bernard Werner Levinger and Richard Steven Varga, On a problem of $O$.

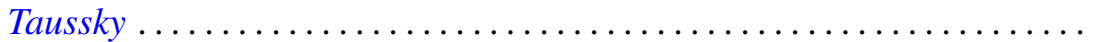

Lowell Duane Loveland, Tame subsets of spheres in $E^{3} \ldots \ldots \ldots \ldots \ldots . .489$

Erik Andrew Schreiner, Modular pairs in orthomodular lattices ......... 519

K. N. Srivastava, On dual series relations involving Laguerre polynomials ...............................

Arthur Steger, Diagonability of idempotent matrices.....

Walter Strauss, On continuity of functions with values in various Banach spaces...

Robert Vermes, On the zeros of a linear combination of polynomials ...

Elliot Carl Weinberg, On the scarcity of lattice-ordered matrix rings ....

Harold Widom, Toeplitz operators on $H_{p} \ldots \ldots \ldots \ldots \ldots$

Neal Zierler, On the lattice of closed subspaces of Hilbert space...

Irving Leonard Glicksberg, Correction to: "Maximal algebras and a theorem of Rado"

John Spurgeon Bradley, Correction to: "Adjoint quasi-differential operators of Euler type"

William Branham Jones, Erratum: "Duality and types of completeness in locally covex spaces".

Stanley P. Gudder, Erratum: "Uniqueness and existence properties of bounded observables" 